\title{
Article
}

\section{SNAP23-Kif5 complex controls mGlu1 receptor trafficking}

\author{
Fabrice Raynaud ${ }^{1,3}$, Vincent Homburger ${ }^{1}$, Martial Seveno ${ }^{2}$, Oana Vigy ${ }^{1}$, Enora Moutin ${ }^{1}$, \\ Laurent Fagni ${ }^{1}$, and Julie Perroy ${ }^{1, \star}$ \\ 1 IGF, CNRS, INSERM, Univ. Montpellier, F-34094 Montpellier, France \\ 2 BioCampus Montpellier, CNRS, INSERM, Univ. Montpellier, F-34094 Montpellier, France \\ 3 Present address: INSERM U1046 PhyMedExp, CNRS UMR9214, Univ. Montpellier, F-34094 Montpellier, France \\ * Correspondence to: Julie Perroy, E-mail: Julie.Perroy@igf.cnrs.fr
}

Edited by Xuebiao Yao

Metabotropic glutamate receptors are expressed at excitatory synapses and control synaptic transmission in mammalian brain. These receptors are involved in numerous patho-physiological functions. However, little is known about the molecular determinants responsible for their intracellular transport and membrane targeting. Here we investigated the nature of the molecular motor and adaptor protein responsible for trafficking and membrane localization of the group I metabotropic glutamate mGlu1 postsynaptic receptor in cultured hippocampal neurons. In proteomic studies, we identified the synaptosome-associated protein 23 (SNAP23) and the molecular motor Kif5 kinesin as proteins interacting with mGlu1 receptor. We showed that SNAP23, but not Kif5, directly interacts with mGlu1 receptor carboxyl terminus. Using a recombination approach to impair or enhance the interaction between SNAP23 and Kif5, we found that the SNAP23-Kif5 complex controls the trafficking of mGlu1 receptor along microtubules. Additional fluorescence recovery after cleavage experiments allowed us to identify a role of the complex in the receptor cell surface targeting. In conclusion, our study indicates that along dendritic processes Kif5-SNAP23 complex contributes to proper mGlu1 receptor trafficking and cell surface expression.

Keywords: mGlu1, SNARE, kinesin, neuron, traffic

\section{Introduction}

Metabotropic glutamate (mGlu1-mGlu8) receptors are G-protein-coupled receptors that are located at brain excitatory synapses to regulate glutamatergic neurotransmission. The mGlu1 and mGlu5 receptor subtypes activate the extracellular signal-regulated kinase (ERK)/mitogen-activated protein kinase (MAPK) pathway, as well as phospholipase $C$, which leads to intracellular calcium release and protein kinase $C$ activation. Regulation of functional expression of mGlu1/5 receptors plays a critical role in brain functions and neurological diseases. For instance, mGlu1 receptor is critically involved in neuronal development (Ichise et al., 2000; Hannan et al., 2001), synaptic plasticity and associated learning and memory (Aiba et al., 1994a, b; Luscher and Huber, 2010), addiction to drugs of abuse (Kenny and Markou, 2004), and several neuropsychiatric and neurodegenerative disorders (Ferraguti et al., 2008). Molecular mechanisms underlying vesicle trafficking of neurotransmitter receptors

Received December 8, 2017. Revised April 9, 2018. Accepted May 11, 2018. (C) The Author(s) (2018). Published by Oxford University Press on behalf of Journal of Molecular Cell Biology, IBCB, SIBS, CAS. All rights reserved. have been of intense interest in the last 10 years, but relatively little is known about the mechanisms controlling the trafficking of mGlu receptors.

Several proteins have been shown to control the functional expression of mGlu1/5 receptors at postsynaptic terminals. These are postsynaptic intracellular scaffolding proteins associated with cytoskeleton, plasma membrane, or fusion proteins. Thus, Homer proteins (Ango et al., 2000), Tamalin (Kitano et al., 2002), Calmodulin (Choi et al., 2011), Neuregulin (Ledonne et al., 2015), VPS26A-SNX27 complex (Lin et al., 2015), and Numb (Zhou et al., 2015) bind to mGlu1 and/or mGlu5 receptors and control their organization in the postsynaptic element and recycling at the synaptic cell surface. This is critical for synaptic plasticity and related cognitive and motor functions. However nothing is known about carrier proteins involved in the longrange delivery of mGlu receptors before their insertion into the postsynaptic membrane.

Soluble N-ethylmaleimide-sensitive-factor Attachment protein REceptor (SNARE) proteins are a class of membrane-associated proteins known to regulate the process of synaptic vesicle fusion with the presynaptic plasma membrane (Lin and Scheller, 
2000; Jahn and Scheller, 2006). However, these proteins are also involved in microtubule-dependent postsynaptic trafficking of glutamate receptors. For instance, SNAP23 is a homolog SNARE protein of SNAP25 (Oyler et al., 1989; Duc and Catsicas, 1995; Tao-Cheng et al., 2000; Washbourne et al., 2002b) that is ubiquitously expressed in neuronal and non-neuronal cells (Ravichandran et al., 1996). In hippocampal neurons, SNAP23 is expressed in both soma and dendrites and regulates the surface expression, recycling, and synaptic function of the ionotropic $\mathrm{N}$-methyl-D-aspartate (NMDA) glutamate receptor (Washbourne et al., 2004; Suh et al., 2010). Other studies have shown that NMDA receptors are transported in clusters along microtubules (Washbourne et al., 2002a) in vesicles containing the kinesin protein Kif17 (Setou et al., 2000; Guillaud et al., 2003). The microtubule-dependent motor Kif5 and the associated kinesin light chain Klc2 would mediate the transport of the alpha-amino3-hydroxy-5-methylisoazol-4-propionate (AMPA) glutamate receptor subunit GLR-1 to the synapse, in Caenorhabditis elegans. This Kif5-dependent transport would control rapid delivery, removal and redistribution of functional postsynaptic AMPA receptors, and thus synaptic strength (Hoerndli et al., 2013).

At present nothing is known about the transport of mGlu receptors along microtubules. Here we report that mGlu1 receptor can form a cargo with SNAP23 in microtubule-associated vesicles conveyed by Kif5 along dendrites of cultured hippocampal neurons. This result provides the first evidence for a molecular mechanism of vesicular transport of postsynaptic mGlu receptors in neurons.

\section{Results}

The Kif5-SNAP23 complex interacts with mGlu1a receptor

In order to identify multiprotein complexes interacting with the intracellular mGlu1a receptor C-terminus, we performed differential proteomic studies in an easily transfectable neuronderived cell line, the $\mathrm{N} 2 \mathrm{a}$ neuroblastoma cell line expressing either full-length YFP-mGlu1a or C-terminus-truncated mGlu1a (YFP-mGlu1a $\Delta \mathrm{C}$ ) receptors. The mGlu1 sequences coverage found using mGlu1a $\Delta \mathrm{C}$ as a bait confirmed the absence of C-terminus mapping (Supplementary Figure S1). Differential analysis of proteins that were immunoprecipitated with these receptors indicated that 173 proteins interact with the fulllength receptor, but not with YFP-mGlu1a $\Delta C$ (Supplementary Table S1). Only proteins with a fold enrichment of six (based on the MSMS ratio) and a minimum of two unique peptides per protein were indeed considered as potential interactors of the carboxyl terminus of the mGlu1 receptor. To determine if these proteins belong to specific physiological categories, we next performed a gene ontology (GO) analysis based on both biological process and cellular component domains. Interestingly, numerous proteins are involved in signaling pathways, transport, and neuronal functions. In search for proteins involved in mGlu1 receptor trafficking, we focused on proteins annotated to the 'intracellular transport' GO term (G0:0046907). Among the 173 potential interactors of mGlu1 C-tail, 29 proteins belong to this functional category, 9 with known protein-protein interaction in the IntAct database (Figure 1). We identified Kif5b (kinesin-1 heavy chain), a molecular motor involved in the vesicular trafficking of AMPA receptors along microtubules (Hoerndli et al., 2013), and SNAP23, a SNARE protein involved in the vesicular transport and exocytosis of various membrane proteins (Lin and Scheller, 2000; Jahn and Scheller, 2006). To address the existence of mGlu1-SNAP23-Kif5b complexes in living cells, we used C6 glioma cells, because the flat shape of these cells was more convenient for microtubule dynamics studies. Moreover, these cells endogenously express mGlu1 receptor (Luis Albasanz et al., 2002; Viwatpinyo and Chongthammakun, 2009; Castillo et al., 2010) and we show that they also express SNAP23 and Kif5 proteins (Figure 2C and D). Immunofluorescent experiments showed that mcherry-tagged mGlu1a receptor colocalized with YFP-tagged Kif5b or YFP-tagged SNAP23 in vesicles, in C6 glioma cell line (Figure 2A and B). Furthermore, YFPtagged mGlu1a receptor could be immunoprecipitated with Kif5 (Figure 2C) and SNAP23 in transfected neuroblastoma cell line (Figure 2D). Altogether these results suggested that mGlu1a receptor can form a complex with Kif5 and SNAP23 in model cell line.

Interaction between mGlu1a receptor and Kif5-SNAP23 complex involves mGlu1a receptor and SNAP23 carboxyl termini

Kif5 and SNAP23 were known to form a complex (Diefenbach et al., 2002), and the above results showed that mGlu1a receptor belongs to the Kif5-SNAP23 complex. We therefore investigated the molecular determinants of the interaction between mGlu1a receptor and Kif5-SNAP23 complex in neuroblastoma cell line. Deletion of the C-terminus of mGlu1a receptor (YFPmGlu1a $\Delta$ C) strongly decreased co-immunoprecipitation of the receptor mutant with both Kif5 and SNAP23 (Figure $2 C$ and D). These experiments showed that the C-terminal domain of mGlu1a receptor was required for the interaction between the receptor and Kif5-SNAP23 complex.

We then investigated whether mGlu1a receptor could directly interact with SNAP23 or Kif5. We measured bioluminescence resonance energy transfer (BRET) signals between these proteins tagged with BRET donor and acceptor compatible entities (mGlu1a-Rluc8 and YFP-SNAP23 or YFP-Kif5). For a constant expression level of mGlu1a-Rluc8, the BRET signal increased hyperbolically as a function of the YFP-SNAP23 expression level, indicating that mGlu1a receptor specifically interacts with SNAP23 (Figure 3A). In contrast, only a weak and linear, non-specific BRET signal could be detected between mGlu1a and Kif5 proteins (Figure 3B). These results suggested that SNAP23 but not Kif5 directly interacts with mGlu1a receptor. This SNAP23 direct interaction could also be detected with mGlu5 but not mGlu7 receptors (Supplementary Figure S2), suggesting that the interaction was specific to group I mGlu receptors (mGlu1a and mGlu5).

We then searched for the interaction domains responsible for the mGlu1a receptor-SNAP23 interaction by measuring BRET signals between C-terminal deletion mutants of these proteins, in transfected neuroblastoma cell line. Successive deletions of the distal part of mGlu1 C-terminus did not affect its BRET 


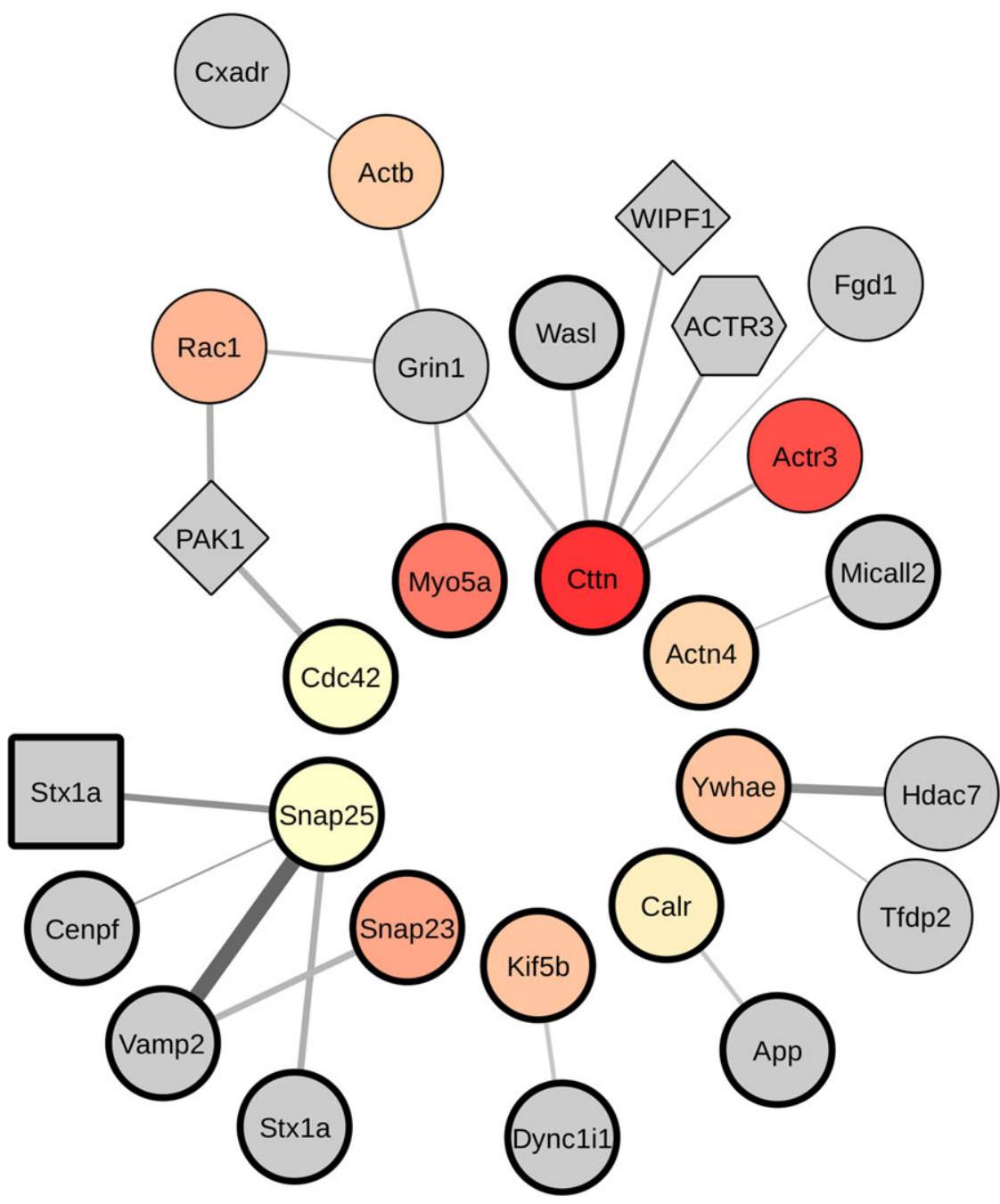

Figure 1 mGlu1a receptor-associated protein network. Searching for proteins involved in mGlu1 receptor trafficking, the network is focused on the nine proteins displayed in the internal circle that are annotated to the 'intracellular transport' GO term and have protein-protein interactions in the IntAct database. It consists of 28 nodes and 22 edges. Node colors reflect the MS/MS ratio and vary from red (high ratio) to light yellow (low ratio). Thick node border highlights proteins annotated to the 'intracellular transport' GO term. Node shapes represent different protein taxonomies: circle (mouse), diamond (human), square (rat), and hexagon (bovine). The edge width is proportional to the number of published manuscripts_curated in the IntAct database-that have reported molecular interaction data. The edge stroke color maps the confidence value: from 0.6 (light gray) to the local maximum 0.92 (dark gray).

signals with wild-type SNAP23 until the receptor lacks all but the 21 proximal amino acids of the tail (mGlu1a $\Delta 341$, Figure 3F). This result showed that interaction with SNAP23 requires the proximal C-terminus of mGlu1a receptor until amino acid position 971 (mGlu1a $\Delta 229$, Figure 3E). BRET signals were also detected between wild-type mGlu1a receptor and SNAP23 mutants deleted from the last 82 C-terminal amino acids, but were lost after deletion of the last $122 \mathrm{C}$-terminal amino acids of the protein (SNAP23 $\Delta 122$, Figure 3 J). These results indicated that interaction of SNAP23 with mGlu1a receptor required the amino acids 88-128 of SNAP23. Altogether these results show that mGlu1a receptor directly interacts with SNAP23 and that this interaction depends on C-terminal domains of both proteins. Thus these results reinforce the above tentative hypothesis that
SNAP23 may bind to Kif5 via its N-terminus (Diefenbach et al., 2002) and serves as a cargo for the transport of mGlu1a receptor by the molecular motor Kif5.

\section{Kif5 controls microtubule trafficking and membrane targeting of the mGlu1a receptor}

Since we found that mGlu1a receptor is part of a Kif5containing complex and because Kif5 is involved in vesicular transport of proteins (Hirokawa et al., 2010), this molecular motor could regulate trafficking along microtubules and cell surface expression of the mGlu1a receptor. We investigated this hypothesis using rapalog-induced reconstruction of Kif5 protein (see Jenkins et al., 2012 for the methodology) in C6 glioma cell line. Briefly, this approach, based on the reconstruction of a 
A

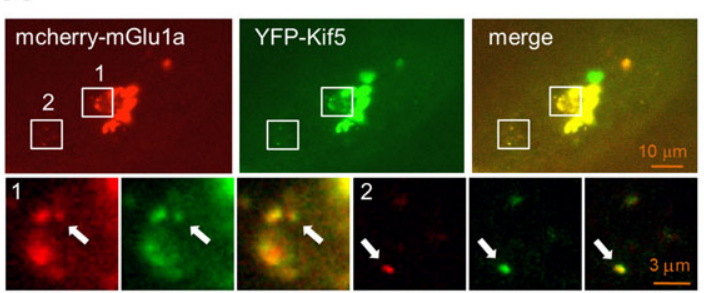

B

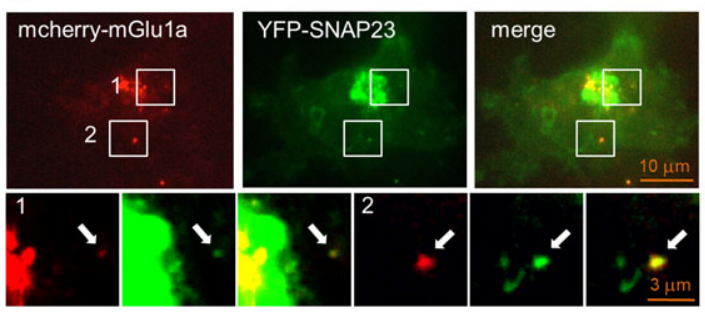

C

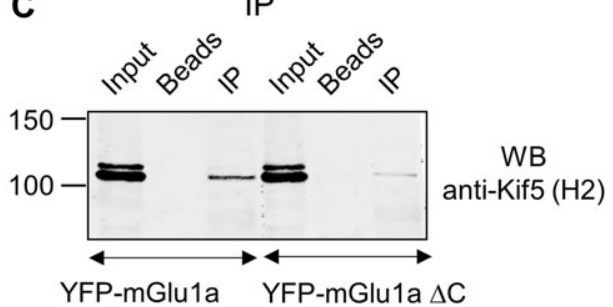

D

IP

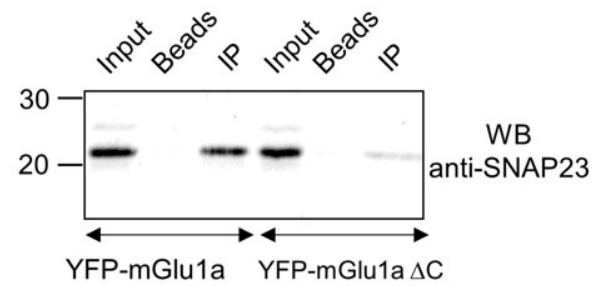

Figure 2 The mGlu1a receptor forms a complex with Kif5b and SNAP proteins. (A and B) Co-localization of mcherry-mGlu1a receptor with YFPKif5 (A) or YFP-SNAP23 (B) proteins in C6 glioma cells. Following a temperature shift at $20^{\circ} \mathrm{C}$ to synchronize post-Golgi traffic and thrombinmediated cleavage of cell surface receptor's mcherry tag, time-lapse images of intracellular protein trafficking were taken at $34^{\circ} \mathrm{C}$ as described in Material and methods section. Arrows indicate mcherry-mGlu1a receptor and YFP-Kif5 (A) or YFP-SNAP23 (B) vesicle co-localization. (C and D) Kif5-SNAP23-mGlu1a receptor complex revealed by co-immunoprecipitation experiments. Neuroblastoma cells were transfected with plasmids encoding wild-type or C-terminus-truncated YFP-mGlu1a receptors and Kif5 (C) or SNAP23 (D) proteins. Cells were washed, solubilized, and subjected to immunoprecipitation (IP) with GFP-trap and revealed by western blots (WB) with either anti-Kif5 (C) or anti-SNAP23 (D) antibodies.

split kinesin by chemical dimerization, enables the identification of motor proteins that interact with specific vesicle populations. To characterize the trapping of SNAP23 and mGlu1 receptor by Kif5 on microtubules, we used the rigor mutant of Kif5, which is stalled on microtubules. Figure 4A shows that mcherry-tagged cargoless-Kif5 recombined with its YFP-tagged cargo binding domain (CBD) decorates microtubules. The YFP-tagged SNAP23 protein colocalized with recombined Kif5 protein on filamentous structures (Figure 4B), suggesting the recruitment of SNAP23 by Kif5 on microtubules. Similarly, forced interaction between SNAP23 and Kif5 using rapalog (Figure $4 \mathrm{C}$ and Supplementary Movie S1) recruited mcherry-tagged mGlu1a receptor to microtubule like structures (Figure 4D). Together, these results suggest that mGlu1a receptor can form a complex with SNAP23 and Kif5 on microtubules, and that SNAP23 may serve as cargo for the transport of mGlu1a receptor by the molecular motor Kif5.

We then investigated whether mGlu1a receptor-Kif5 interaction could control membrane targeting of the receptor. The mGlu1a receptor was tagged with an extracellular-thrombin cleavable YFP tag and transfected in C6 glioma cells. Following thrombininduced extinction of cell surface receptor fluorescence, we could measure intracellular receptor trafficking efficiency and kinetics from the Golgi to the cell surface, by fluorescence recovery after cleavage (FRAC) experiments. Interestingly, deletion of the C-terminus of mGlu1a receptor (YFP-th/mGlu1a $\Delta \mathrm{C}$ ) significantly delayed plasma membrane targeting of the mutant receptor over a period of $120 \mathrm{~min}$ (Figure 5A). Knowing that the C-terminus of mGlu1a is required for interaction of the receptor to the Kif5-containing complex (Figure 3), these experiments suggested that mGlu1a receptor association with Kif5 complex is essential for the receptor plasma membrane targeting.

We further investigated this hypothesis by measuring the dominant-negative effects of two Kif5 mutants, Kif5 rigor and Kif5 unbound, in C6 glioma cell line. Kif5 rigor is an active form of Kif5 that binds to microtubules and blocks microtubuledependent protein transport. This mutant inhibited cell surface expression of YFP-tagged mGlu1a receptor (Figure 5B), suggesting that membrane expression of the receptor involves microtubule-dependent vesicular traffic of proteins. The Kif5 unbound mutant is an inactive form of Kif5 that lacks ability to interact with microtubules and therefore aborts the transport of Kif5-specific cargo. This mutant also inhibited membrane expression of YFP-tagged mGlu1a receptor (Figure 5B), thus suggesting involvement of Kif5 in membrane trafficking of the receptor. We then investigated the specificity of the effect of Kif5 on mGlu1a receptor membrane trafficking by comparing the effects of Kif5 and Kif3 unbound mutants on membrane expression of YFPtagged mGlu1a receptor in FRAC experiments. Only Kif5 mutant significantly inhibited mGlu1a receptor plasma membrane recovery, $1 \mathrm{~h}$ after cleavage of the receptor extracellular YFP tag with thrombin (Figure $5 \mathrm{C}$ ). We also tested the effects of Kif5 rigor and Kif5 unbound mutants on cell surface targeting of N-terminal YFP-tagged mGlu7 receptor in FRAC experiments. Unlike YFP-tagged mGlu1 receptors, the membrane targeting of YFP-mGlu7 receptor was unaffected by Kif5 mutants (Figure 5D). These experiments suggested that Kif5 controls membrane targeting of mGlu1a receptor via microtubule-dependent transport in 66 glioma cell line. 
A mGlu1a-Rluc8 + YFP-SNAP23

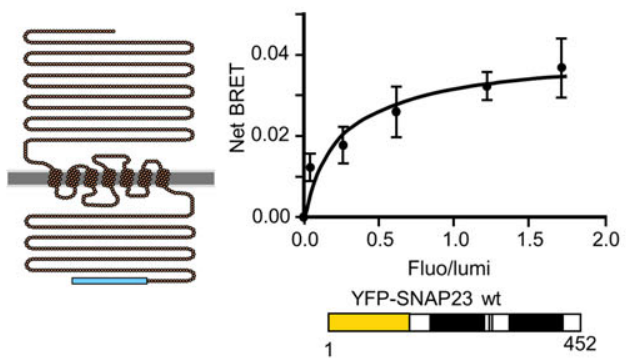

C

mGlu1a $\Delta 62$-Rluc8 + YFP-SNAP23
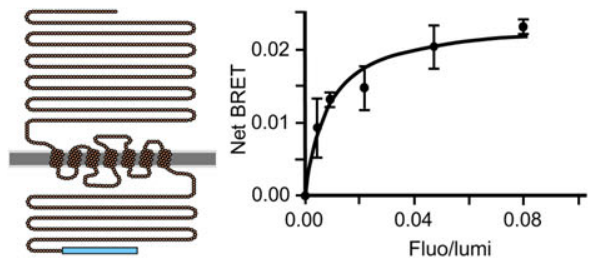

D mGlu1a $\triangle 171-$ Rluc8 + YFP-SNAP23
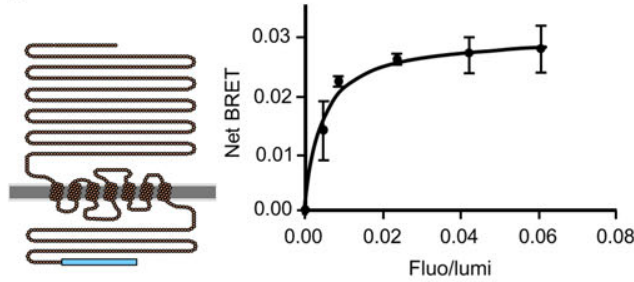

E mGlu1a $\Delta 229-$ Rluc8 + YFP-SNAP23
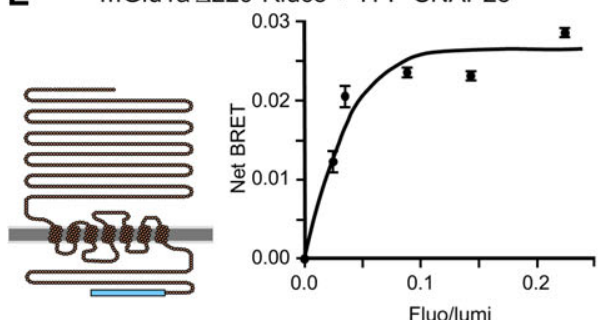

$\mathbf{F}$

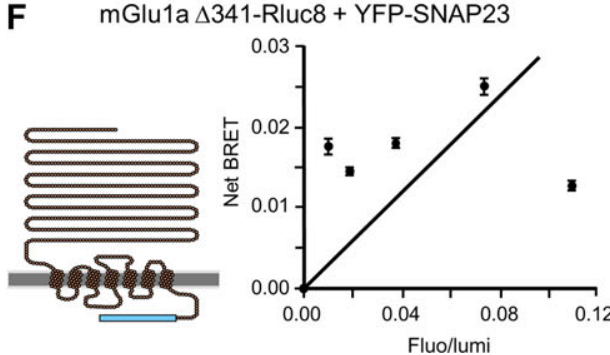

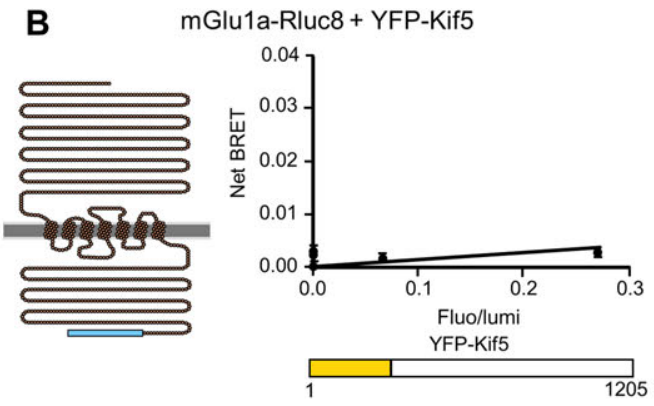

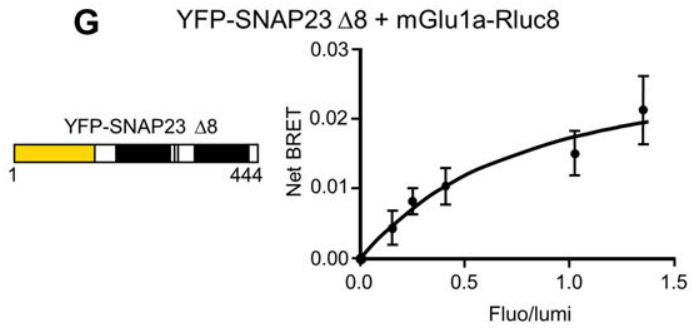

H YFP-SNAP23 $\triangle 49+$ mGlu1a-Rluc8

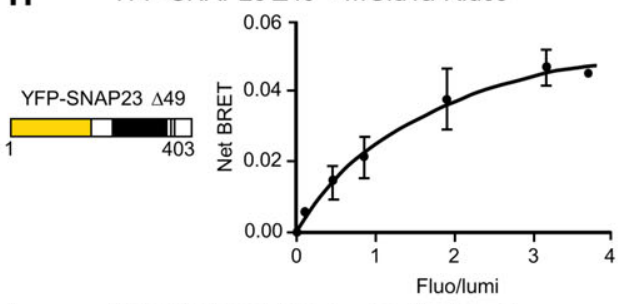

I

YFP-SNAP23 $\Delta 82+$ mGlu1a-Rluc8

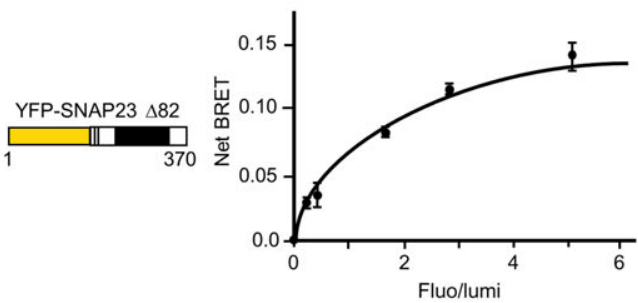

J YFP-SNAP23 $\Delta 122+$ mGlu1a-Rluc8

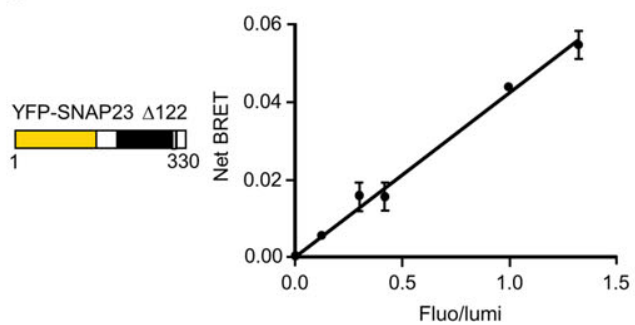

Figure 3 Identification of mGlu1a receptor and SNAP23 interaction domains by BRET analysis. (A and B) mGlu1a directly interacts with SNAP23, but not Kif5. BRET signal between mGlu1a-Rluc8 and YFP-SNAP23 (A) or mGlu1a-Rluc8 and YFP-Kif5 (B) constructs. Neuroblastoma cells were co-transfected with a fixed concentration of mGlu1a-Rluc8 and increasing concentrations of YFP-SNAP23 (A) or YFP-Kif5 (B). (C-F) mGlu1a receptor proximal C-terminus (until amino acid position 971) is required for its interaction with SNAP23. BRET signal between truncated mGlu1a-Rluc8 receptors and YFP-SNAP23. Neuroblastoma cells were co-transfected with a fixed concentration of C-terminus-truncated mGlu1a receptor fused to Rluc8 constructs as illustrated, and increasing concentrations of YFP-SNAP23 plasmids. (G-J) SNAP-23 amino acids 88-128 are required for its interaction with mGlu1a receptor. BRET signal between mGlu1a-Rluc8 receptors and C-terminus-truncated YFP-SNAP23 proteins. Neuroblastoma cells were co-transfected with a fixed concentration of Rluc8-tagged mGlu1a receptor and increasing concentrations of YFP-tagged truncated SNAP23 mutants as illustrated. The data shown are representatives of five independent experiments performed in triplicate and expressed as mean \pm SEM. The curves were fitted using a nonlinear regression equation, assuming a single binding site. Note that mGlu1a-Rluc8 receptor did not interact with YFP-Kif5 (B), mGlu1a $\Delta 341$ receptor mutant did not interact with YFP-SNAP23 (F), and YFP-SNAP23 122 mutant did not interact with mGlu1a-Rluc8 receptor (J), as indicated by a BRET signal that increased linearly (rather than hyperbolically) with the YFP-tagged protein expression level, which most likely reflected random collision. 


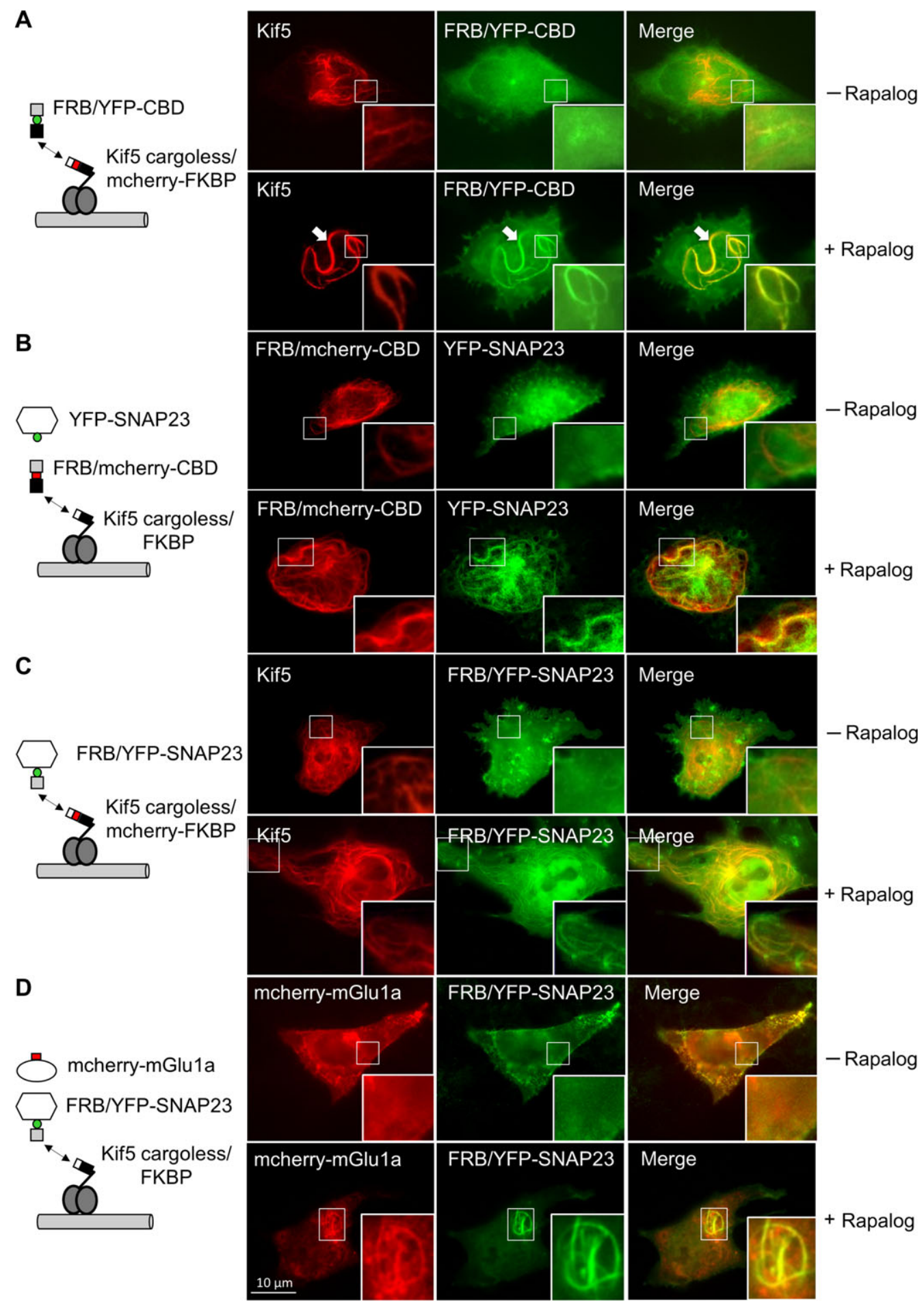

Figure 4 Kif5-SNAP23 complex controls microtubule trafficking of the mGlu1a receptor. Visualization of Kif5-SNAP23-mGlu1a receptor complex after rapalog treatment in $\mathrm{C} 6$ glioma cell line. Images were obtained in the absence (upper line) or presence (bottom line) of the rapalog dimerizer. The framed area is magnified in the right bottom corner. (A) Kif5 rigor-stained microtubules after rapalog-induced reconstruction of split Kif5 (arrows). Cells were transfected with Kif5 cargoless/mcherry-FKBP and YFP-tagged rapamycin-binding domain (FRB) associated with kinesin CBD (FRB/YFP-CBD) constructs. (B) SNAP23-stained microtubules after rapalog-induced Kif5 rigor reconstruction (arrows). Cells were transfected with split Kif5 cargoless/FKBP, FRB/mcherry-CBD, and YFP-SNAP23 constructs. (C) YFP-SNAP23-decorated microtubules after rapalog-induced forced interactions between SNAP23 and Kif5. Cells were transfected with Kif5 rigor cargoless/mcherry-FKBP and FRB/YFP-SNAP23 constructs. (D) mcherry-mGlu1a-stained microtubules induced by rapalog-forced interactions between SNAP23 and Kif5. Cells were transfected with the split Kif5 rigor cargoless/FKBP, FRB/YFP-SNAP23, and mcherry-mGlu1a receptor constructs. 


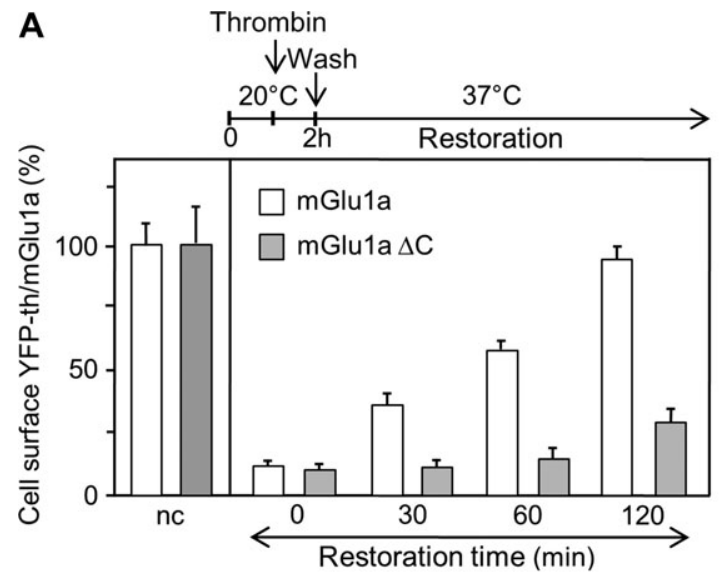

C

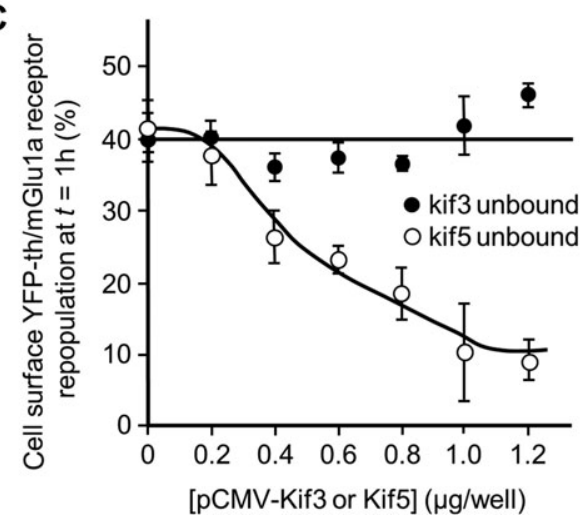

B

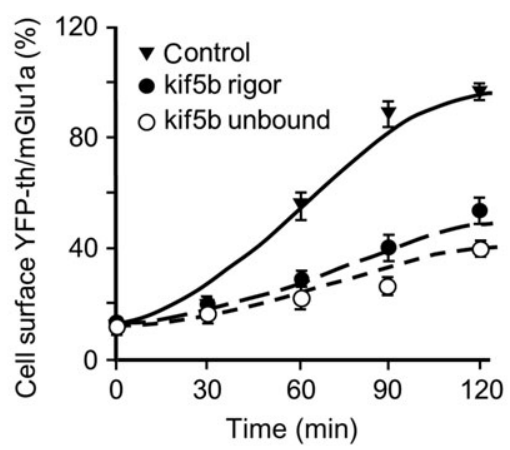

D

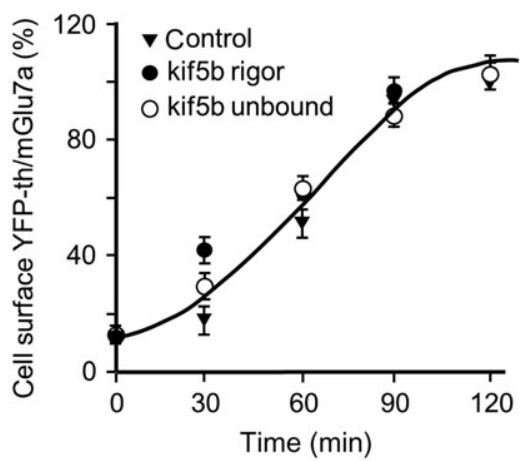

Figure 5 Kif5 is involved in mGlu1a receptor post-Golgi traffic. (A) mGlu1a receptor cell surface targeting depends on the receptor C-terminus. Schematic view of FRAC experiments (upper part). YFP-th/mGlu1a wild-type receptor (YFP-th/mGlu1a) or C-terminus-truncated receptor (YFP-th/mGlu1a $\Delta \mathrm{C}$ ) constructs were transfected in $\mathrm{C} 6$ glioma cells. Cell surface recovery of each receptor construct was expressed at different time points after thrombin-induced cleavage of the extracellular YFP tag and expressed as percentage of control wild-type receptor surface expression obtained in the absence of thrombin treatment (nc white bar). For this and following panels, values are mean \pm SEM of three independent experiments. (B) Negative kif5 mutants inhibit cell surface mGlu1a receptor targeting. C6 glioma cells were cotransfected with YFP-th/mGlu1a receptor and kif5 rigor or kif5 unbound mutant constructs and subjected to FRAC experiments (same as in A). Results indicate a 50\% inhibition of maximal cell surface receptor recovery in the presence of dominant-negative kif5 mutants. (C) Doseresponse relationship and kif5-specific inhibition of cell surface YFP-th/mGlu1a receptor targeting. C6 glioma cells were co-transfected with a fixed concentration of YFP-th/mGlu1a receptor construct and increasing concentrations of dominant-negative Kif5 or Kif3 unbound mutant constructs. Cells were then subjected to FRAC experiments to measure cell surface recovery of YFP-th/mGlu1a receptor $1 \mathrm{~h}$ after cleavage of the YFP tag with thrombin (same as in A). Results indicate inhibition of cell surface receptor recovery with increasing concentrations of Kif5, but not Kif3 unbound constructs. (D) Dominant-negative Kif5 mutants did not modify mGlu7a receptor cell surface expression. Cells were cotransfected with YFP-th/mGlu7a receptor and Kif5 rigor or Kif5 unbound mutants and subjected to FRAC experiments to measure cell surface YFP-th/mGlu7a receptor recovery after cleavage of its external YFP tag (same as in A). Results indicate no modification in receptor recovery in the presence of dominant-negative Kif5 rigor and unbound mutants.

\section{The Kif5-SNAP23 complex controls mGlu1a receptor membrane targeting in neurons}

We then investigated whether the role of Kif5-SNAP23 complex in membrane targeting of mGlu1a receptor that we found in C6 glioma cell line also applied to neurons. Transfected mcherry-tagged mGlu1a receptor colocalized with YFP-tagged Kif5 or YFP-tagged SNAP23 in mouse cultured hippocampal neurons (Figure 6A and B; Supplementary Movies S2-S4). These results suggest that the Kif5-SNAP23 complex is involved in the vesicular transport of mGlu1a receptor in neurons. We then investigated the role of this complex in mGlu1a receptor membrane targeting. Deletion of the C-terminus of mGlu1a receptor (YFP-th/mGlu1a $\Delta$ C, Figure 7A) delayed membrane targeting of YFP-tagged mGlu1a receptor in neurons, indicating a role of the C-terminus of the receptor in this process. As Kif5 was found to control membrane targeting of mGlu1a receptor in $\mathrm{C} 6$ glioma cells (Figure 5), we investigated whether this also applied to neurons. We found that Kif5 unbound mutants altered membrane recovery of YFP-tagged mGlu1a receptor (Figure 7B) in FRAC experiments, in neurons. Conversely, deletion of postsynaptic density protein, 
A

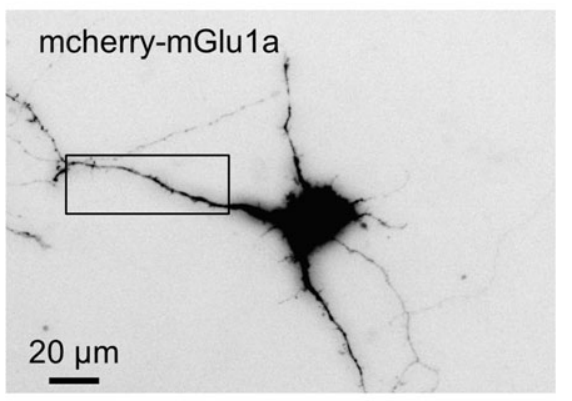

mcherry-mGlu1a

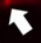
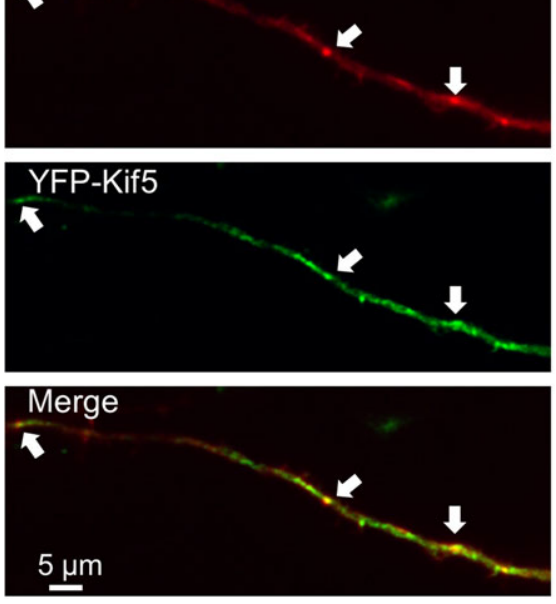

B
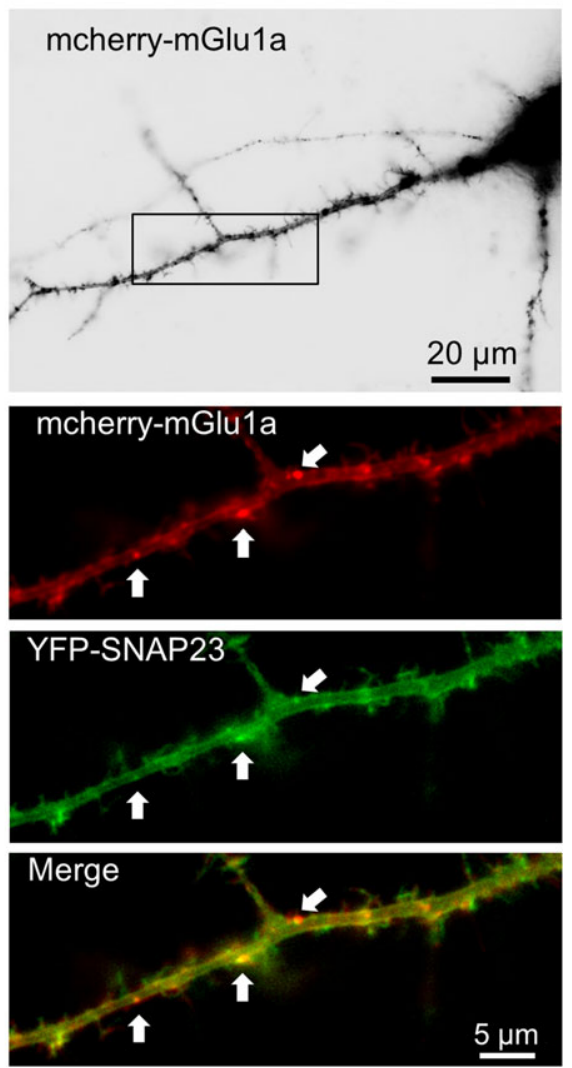

Figure 6 Co-localization of mcherry-mGlu1a receptor with Kif5 and SNAP23 proteins in hippocampal neurons. Arrows indicate co-localization of mcherry-mGlu1a receptor with Kif5 (A) and SNAP23 (B).

Drosophila disc large tumor suppressor, zonula occludens-1 protein (PDZ) and homer interaction motifs included in the C-terminus of mGlu1a receptor did not affect the membrane targeting of the receptor (Figure $7 \mathrm{C}$ ). Together these experiments suggest that the molecular motor Kif5 binds to the cargo protein SNAP23 and recruits mGlu1a receptors to control the receptor membrane targeting in neurons.

\section{Discussion}

Here we revealed aspects of the molecular mechanisms of postsynaptic mGlu1 receptor transport in neurons. We first identified Kif5 and SNAP23 as molecular motor and adaptor protein associated with mGlu1 receptor. We show that SNAP23-Kif5 complex controls mGlu1 receptor dendritic trafficking via microtubule-dependent transport. Previous studies have shown that the molecular motor myosin VI and Kif17 (Wu et al., 2002), and vesicular fusion protein SNAP23 and SNAP25 (Osten et al., 1998; Noel et al., 1999; Washbourne et al., 2004; Suh et al., 2010) regulate synaptic delivery of ionotropic AMPA and NMDA glutamate receptors. Thus the present and previous results further argue in favor of an important role of kinesins and SNARE proteins in the postsynaptic delivery of glutamate receptor family, therefore participating to the formation and possibly maturation of glutamatergic synapses.
Our study was focused on mGlu1 receptor trafficking along dendrites, because we found that mGlu1 receptor in cultured mouse hippocampal neurons is only expressed in dendrites (data not shown; Das and Banker, 2006). However, in other cell types, mGlu1 receptors can be expressed at presynaptic sites as well. The nature of molecular motors and adaptors involved in the presynaptic trafficking of mGlu1 receptors is still unknown. Interaction with other protein partners might trigger specific subcellular sorting and trafficking of the receptor.

A proteomic approach following mGlu1 receptor immunoprecipitation experiments allowed us to identify a large number of proteins including nine proteins known to be involved in cellular transport (Figure 1). Among these proteins, we focused on Kif5 and SNAP23. Kif5 and SNAP23 were known to form a complex (Diefenbach et al., 2002). Here we corroborated this result and further showed that Kif5-SNAP23 complex could in turn interact with the carboxyl terminus of mGlu1 receptor thus forming a Kif5-SNAP23-mGlu1 receptor complex. We found that this complex was recruited on microtubule like structures, which was consistent with the hypothesis that the molecular motor Kif5 could be responsible for the vesicular transport of the SNAP23-mGlu1 receptor cargo along dendritic microtubules. As SNAP23 belongs to the large family of SNARE proteins involved in membrane fusion processes, SNAP23 may also control exocytosis of the 


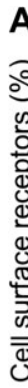

A

A

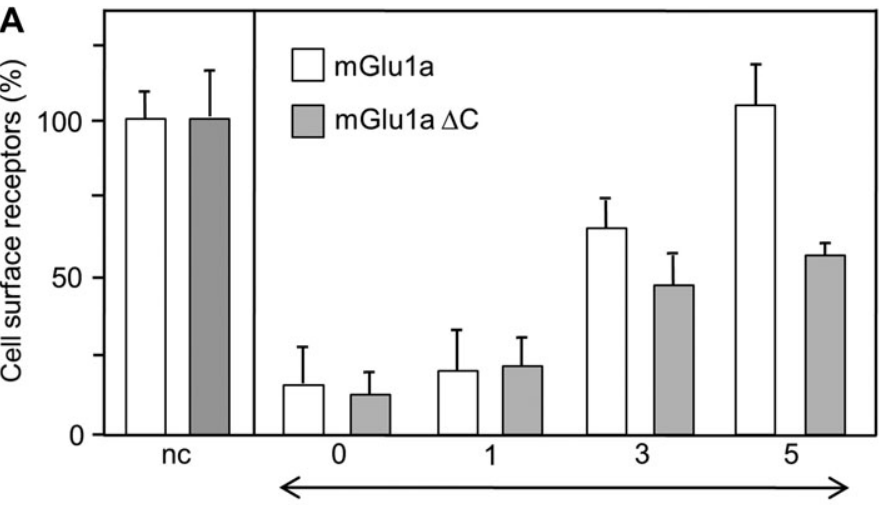

Restoration time (h)

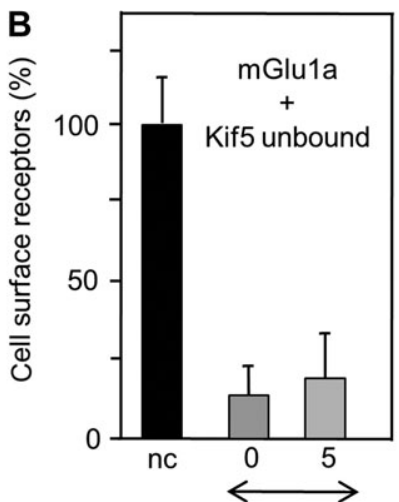

Restoration time $(\mathrm{h})$

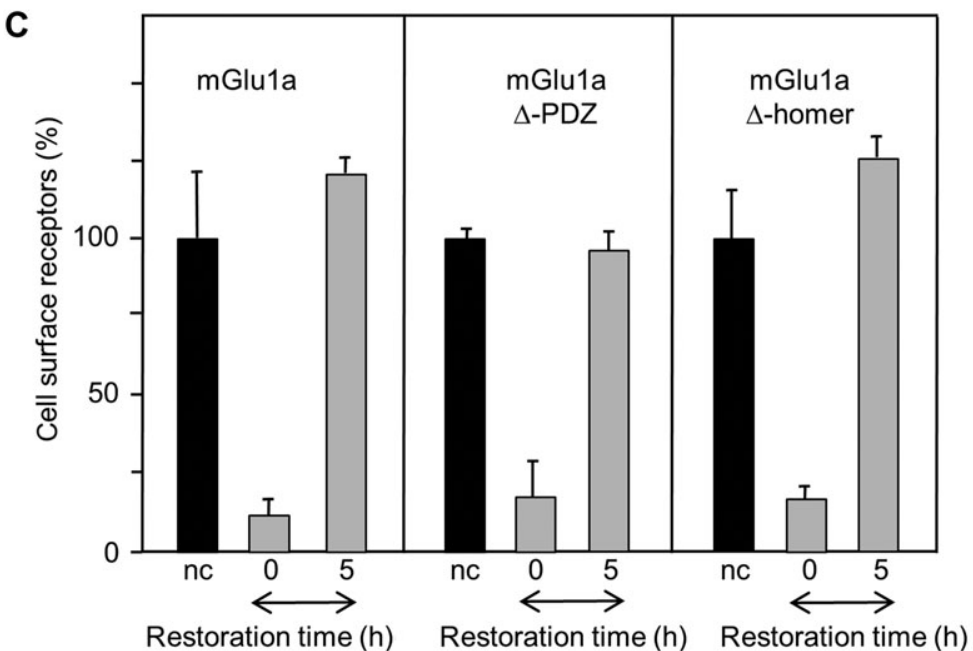

Figure 7 mGlu1a receptor C-terminus- and Kif5-dependent cell surface targeting of YFP-th/mGlu1a receptor in cultured hippocampal neurons. (A) Deletion of YFP-th/mGlu1 receptor C-terminus impairs cell surface targeting of YFP-th/mGlu1a receptor. Wild-type YFP-mGlu1a receptor (mGlu1a) or C-terminus-truncated receptor (mGlu1a $\Delta \mathrm{C}$ ) constructs were transfected in hippocampal neurons (same as Figure $5 \mathrm{~A}$ ). For this and following panels, values are mean \pm SEM of three independent experiments. (B) Kif5 unbound mutant prevents YFP-th/mGlu1 receptor cell surface recovery. FRAC experiments performed in neurons co-transfected with wild-type YFP-th/mGlu1a receptor (mGlu1a) and Kif5 unbound mutant. (C) PDZ and homer interaction motifs are not involved in cell surface YFP-th/mGlu1a receptor targeting. Wild-type YFP-mGlu1a receptor (mGlu1a) or receptor deleted from its PDZ (mGlu1a $\Delta$-PDZ) or homer (mGlu1 $\Delta$-homer) binding motif was transfected in hippocampal neurons. Cells were then processed as in $\mathbf{B}$.

mGlu1 receptor to the cell surface. This hypothesis was consistent with our finding that the deletion of the carboxyl terminus of mGlu1 receptor delayed the membrane targeting of the receptor. The existence of a Kif5-SNAP23-mGlu5 complex suggests that Kif5-SNAP23-mediated transport could apply to both mGlu1 and mGlu5 receptors.

SNARE proteins mediate membrane fusion events in various cell types (Lin and Scheller, 2000; Jahn and Scheller, 2006). For instance, SNAP25 is a neuron-specific SNARE protein that regulates synaptic vesicle fusion with the presynaptic plasma membrane (Lin and Scheller, 2000; Jahn and Scheller, 2006), and membrane delivery of postsynaptic AMPA receptors (Hoerndli et al., 2013). Postsynaptic translocation of AMPA receptors has also been shown to depend on the actin motor myosin $\mathrm{VI}$ and the adaptor protein SAP97 (Wu et al., 2002). The SNAP25 homolog, SNAP23, is ubiquitously expressed in neuronal and non- neuronal cells. In the brain, SNAP23 and SNAP25 are both located in the somato-dendritic neuronal compartment, but display non-overlapping distribution. SNAP23 mediates the membrane delivery of NMDA receptors (Suh et al., 2010). Here we show that SNAP23 regulates the dendritic trafficking and postsynaptic membrane delivery of mGlu1 receptors. Thus consistent with the somato-dendritic location of SNAP23, both mGlu1 and NMDA receptors bind to SNAP23. However, these receptors seem to display distinct vesicular transport. The NMDA receptor was shown to be transported by the molecular motor Kif17 (Setou et al., 2000; Guillaud et al., 2003). Whether SNAP23 and Kif17 belong to the same complex to transport NMDA receptor is still an open question. If so, this would imply that mGlu1 and NMDA receptors are sorted to two different motors (Kif5 and Kif17) through the same adaptor. An interesting topic would then be to understand which molecular determinant beside the 
adaptor itself could specify the interaction with selective molecular motors, such as specific cargo-adaptor conformation or post-translational modifications. An attractive alternative hypothesis would be that adaptors would specify the nature of the molecular motor, which can be supported by the fact that mLin-10 has been identified as adaptor protein for Kif17 in the transport of NMDA receptors (Setou et al., 2000). Hence more explorations are needed to fully understand these molecular mechanisms, but Kif5, Kif17, and myosin VI molecular motors as well as SAP97, SNAP23, and SNAP25 proteins seem to play essential roles in glutamate receptors specific trafficking.

Metabotropic glutamate receptors modulate efficacy of synaptic transmission mediated by the ionotropic AMPA and NMDA glutamate receptors. Regulation of mGlu receptors delivery at postsynaptic sites is essential for an optimal functioning of the synapse. The strength of synaptic transmission also depends on the number of functional postsynaptic ionotropic AMPA and NMDA glutamate receptors. For instance, long-term potentiation and long-term depression of synaptic transmission can result from an increased and decreased number of active postsynaptic ionotropic glutamate receptors, respectively. Parallel modification in the number of mGlu receptors should therefore be required to maintain an optimal tuning of either the potentiated or depressed synapse. Thus kinesins and SNARE proteins would play essential roles not only during synaptogenesis, but also during synaptic plasticity by controlling appropriate delivery of metabotropic receptors to maintain adequate synaptic tuning. It is striking that glutamate receptors use different proteins for their intracellular trafficking although they may be translocated to same postsynaptic sites. One possible reason for such different mechanisms is that each receptor may need specific regulation for appropriate tuning of the synapse. Therefore, this implies fine regulation of the glutamate receptor assembly with their cargo proteins, a process that remains largely elusive currently.

\section{Materials and methods}

\section{DNA constructs}

YFP-mGlu1a, mGlu1a $\Delta$ C (equivalent to mGlu1a $\Delta 341$ ), mGlu1aYFP, and mGlu7a plasmids were gifts from Dr Jean-Philippe Pin (IGF, CNRS, INSERM, Université Montpellier).

The plasmid YFPth-mGlu1a was obtained by introducing the thrombin cleavage sequence GGLVPRGSGG immediately after the YFP sequence by site-directed mutagenesis using oligonucleotide: 5'-ATACACGCGTACCACCACTGCCTCTCGGAACTAAACCACCCTTGTACA GCTCGTCCAT-3' and subcloning. The plasmid mcherry-th-mGlu1a was obtained by exchanging YFP for mcherry. The plasmid p-YFP-th-mGlu1a $\Delta C$ was obtained by introducing YFP-th in p-mGlu1a $\Delta$ C. The plasmid p-mGlu1a-Rluc8 was obtained by exchanging YFP from $p$-mGlu1a-YFP for Rluc8 from pcDNA-Rluc8. The plasmid $p$-YFP-th-mGlu1a $\Delta$ PDZ was obtained by mutation of PDZ binding motif (STL) in p-YFP-th-mGlu1a. The plasmid p-YFP-th-mGlu1a $\Delta$ homer was obtained by mutation of homer binding motif (PPSPFR) in p-YFP-th-mGlu1a. The plasmids p-mGlu1a- $\Delta 341$-Rluc8, p-mGlu1a- $\Delta 229$-Rluc8, p-mGlu1a- $\Delta 171$ -
Rluc8, and p-mGlu1a- $\Delta 62$-Rluc8 were obtained by inserting Rluc8 ended by a stop codon after positions $859,971,1029$, and 1138 of p-mGlu1a coding sequence (1200 amino acids). In the plasmids p-mGlu1a- $\Delta 229$-Rluc8, p-mGlu1a- $\Delta 171-$ Rluc8, and p-mGlu1a- $\Delta 62-$ Rluc8, the retention motif RRKK in the C-terminal coding sequence of mGlu1 was exchanged for AAAA to avoid endoplasmic reticulum retention as previously described for this receptor (Chan et al., 2001).

The plasmid YFP-th-mGlu7a was obtained by introducing YFP-th before the coding sequence of $\mathrm{p}$-mGlu7a.

Kif5b and Kif5c constructs were generated from the mouse Kif5b and mouse Kif5c IMAGE clones (\#30543821 and \#30536079, respectively; Gene Service, Source BioScience). The rigor mutation was obtained using point mutation-containing primers to substitute the threonine 92 by a valine. We created the unbound mutation using point mutation-containing primers to substitute the serine 204 by a glycine and the histidine 205 by a glycine. The plasmids $p$-YFP-Kif5 $b$ and p-YFP-Kif $5 c$ were obtained by PCR amplification of the EYFP coding sequence from $p$-EYFP and insertion into $\mathrm{p}$-Kif5b and $\mathrm{p}$-Kif5c.

To obtain p-FRB-YFP-CBD, we amplified by PCR the Kif5b coding sequence (amino acids 816-964) from p-Kif5b and inserted into p-FRB-YFP-snap23. The sequence 816-964 contains the CBD and the minimal domain for SNAP23 binding (814-907) as previously described (Diefenbach et al., 2002).

p-Kif5 rigor cargoless-mcherry was obtained by introducing mcherry after position 560 in Kif5c coding sequence. This plasmid was further transformed into $\mathrm{p}$-Kif5 rigor cargoless-mcherryFKBP using $\mathrm{pC}_{4} \mathrm{M}-\mathrm{F} 2 \mathrm{E}$ (ARIAD Pharmaceuticals, Inc); and p-Kif5 rigor cargoless-FKBP was obtained by mcherry coding sequence removal.

p-Kif3a was generated from the human Kif3a IMAGE clone (\#5298675; Gene Service, Source BioScience). From p-Kif3a, we created the unbound mutation using point mutation-containing primers to substitute the serine 220 by an alanine and the histidine 221 by an alanine.

SNAP23 constructs were generated with the mouse SNAP23b IMAGE clone (\#30606358; Gene Service, Source BioScience). The plasmid p-YFP-SNAP23 was obtained by subcloning the PCR amplified SNAP23 coding sequence (210 amino acids) into p-EYFP (Clontech); p-YFP-SNAP23 $\Delta 8, \Delta 49, \Delta 82$, and $\Delta 122$ were obtained by introducing a stop codon after positions 202, 161, 128 , and 88 of the SNAP23b coding sequence.

The plasmid $p$-FRB-YFP-SNAP23 was obtained by insertion of the $F R B$ coding sequence from $\mathrm{pC}_{4}-\mathrm{R}_{\mathrm{H}} \mathrm{E}$ (ARIAD Pharmaceuticals, Inc.) into $p$-YFP-SNAP23, after introduction of a Spe1 restriction site just after the initiation codon in p-YFP-SNAP23. All mutants were generated by PCR and verified by sequencing.

\section{Neuroblastoma cells, C6 glioma cells, and hipppocampal neuronal culture}

Neuroblastoma N2a cells were obtained from $\mathrm{Dr}$ A. Varrault (IGF, Montpellier). They were cultured as previously described (Brabet et al., 1991). Cells were transfected with lipofectamine 2000 (Invitrogen) according to the manufacturer's instructions. 
C6 glioma cells were cultured and transfected as described previously (Giau et al., 2005). Wistar rat hippocampal cultures were prepared from E-17/18 embryos of either sex and grown in Neurobasal medium supplemented with $0.5 \mathrm{mM}$ L-glutamine, $2 \%$ B27, and $10 \%$ heat-inactivated horse serum (all from Invitrogen). Hippocampal neurons were transfected at DIV-9 with lipofectamine 2000 (Invitrogen) according to the manufacturer's standard protocol. For immunofluorescence and FRAC experiments, cell lines were plated onto coverslips (12 or $35 \mathrm{~mm}$ diameter) and hippocampal neurons onto coverslips coated with $50 \mu \mathrm{g} / \mathrm{ml}$ poly-D-lysine (Sigma).

\section{Protein separation and identification by LC-MS/MS}

YFP-mGlu1a or YFP-mGlu1a $\Delta C$ receptors immunoprecipitated with GFP-Trap-A were separated on SDS-PAGE gels (12\% polyacrylamide, Mini-PROTEAN ${ }^{\circledR}$ TGXTM Precast Gels, Bio-Rad) and stained with Protein Staining Solution (Euromedex). Gel lanes were cut into five gel pieces and destained with $50 \mathrm{mM}$ triethylammonium biCarbonate (TEABC) and three washes in 100\% acetonitrile. Proteins were digested in gel using trypsin (500 ng/band, Gold, Promega) as previously described (Thouvenot et al., 2008). Digest products were dehydrated in a vacuum centrifuge and reduced to $3 \mu \mathrm{l}$. The generated peptides were analyzed online by nanoflowHPLC-nanoelectrospray ionization using on a LTQ-Orbitrap XL mass spectrometer (Thermo Scientific) coupled to an Ultimate 3000 HPLC (Thermo Fisher Scientific). Desalting and pre-concentration of samples were performed online on a Pepmap ${ }^{\circledR}$ pre-column (0.3 $\mathrm{mm} \times 10 \mathrm{~mm}$, Dionex). A gradient consisting of $0-40 \% \mathrm{~B}$ for $60 \mathrm{~min}$ and $80 \% \mathrm{~B}$ for $15 \mathrm{~min}(A=0.1 \%$ formic acid, $2 \%$ acetonitrile in water; $B=0.1 \%$ formic acid in acetonitrile) at 300 $\mathrm{nl} / \mathrm{min}$ was used to elute peptides from the capillary reversephase column $\left(0.075 \mathrm{~mm} \times 150 \mathrm{~mm}\right.$, Acclaim Pepmap $100^{\circledR}$ C18, Thermo Fisher Scientific). Eluted peptides were electrosprayed online at a voltage of $2.4 \mathrm{kV}$ into a LTQ-Orbitrap XL mass spectrometer. A cycle of one full scan mass spectrum $(400-2000 \mathrm{~m} / \mathrm{z}$ ) at a resolution of 60000 (at $400 \mathrm{~m} / \mathrm{z}$ ), followed by five data-dependent MS/MS spectra was repeated continuously throughout the nanoLC separation. All MS/MS spectra were recorded using normalized collision energy (35\%, activation) with an isolation window of $3 \mathrm{~m} / \mathrm{z}$. Data were acquired using the Xcalibur software ( $v$ 2.0.7). For all full scan measurements with the Orbitrap detector, a lock-mass ion from ambient air ( $m / z$ 445.120024) was used as an internal calibrant as described (Olsen et al., 2005). Analysis of MS data was performed using the MaxQuant software package ( $v$ 1.5.5.1) (Cox and Mann, 2008). Tandem mass spectra (MS/MS) were searched by the Andromeda search engine (Cox et al., 2011) against the UniProtKB Reference Proteome UP000000589 database for the Mus musculus taxonomy (release 2017_08, 22277 canonical entries) and the specific sequence of interest using the following parameters: enzyme specificity was set as Trypsin/P, and a maximum of two missed cleavages and a mass tolerance of $0.5 \mathrm{Da}$ for fragment ion were applied. A second database of known contaminants provided with the MaxQuant suite was also employed. The 'match between runs' and 'iBAQ' options were checked. Oxidation
(M) was specified as variable modification. Database searches were performed with a mass tolerance of $20 \mathrm{ppm}$ for precursor ion for mass calibration, and with a 4.5-ppm tolerance after calibration. The maximum false peptide and protein discovery rate was specified as 0.01 . Seven amino acids were required as minimum peptide length. The MaxQuant software generates several output files that contain information about identified peptides and proteins. The 'proteinGroups.txt' file is dedicated to the identified proteins: each single row collapses into protein groups all proteins that cannot be distinguished based on identified peptides. An in-house bioinformatics tool has been developed to automatically select a representative protein ID in each protein group. First, proteins with the most identified peptides are isolated in a so called 'match group' (proteins from the 'Protein IDs' column with the maximum number of 'peptides counts (all)'). For the remaining match groups where more than one protein ID existed after filtering, the 'leading' protein has been chosen as the best annotated protein in UniProtKB (reviewed entries rather than automatic ones, the one with the highest protein existence evidence or the most annotated protein according to the number of GO annotations dated from 05/09/2017).

\section{Bioinformatics and network analysis}

Only proteins with a fold enrichment of six (based on the MS/MS ratio) and a minimum of two unique peptides per protein were considered as potential interactors of the carboxyl terminus of the mGlu1 receptor (Supplementary Table S1). Protein-protein interactions of these 173 proteins have been downloaded from the IntAct database (Orchard et al., 2014) (October 12, 2017) and filtered based on an edge score set to 0.6 (high confidence). Protein network analysis was performed using Cytoscape (Shannon et al., 2003) (version 3.5.1) and the ClueGO application (Bindea et al., 2009) (v2.3.4) was used to handle GO annotations (from EBIQuickGO-GOA, September 6-October 16, 2017). A focus was made on proteins annotated to the 'intracellular transport' $\mathrm{GO}$ term, GO:0046907 (29/173 proteins; 9/29 with known PPI in IntAct).

\section{Immunoprecipitation and western blots}

Neuroblastoma cells were transfected with YFP-mGlu1a or C-terminus-truncated YFP-mGlu1a (YFP-mGlu1a $\Delta \mathrm{C}$ ) receptor. Twenty-four hours after transfection, cells $(3 \times 10-\mathrm{cm}$ diameter plates per condition) were washed and subjected to $20^{\circ} \mathrm{C}$ temperature blockade for $2 \mathrm{~h}$ without thrombin and then shifted to $37^{\circ} \mathrm{C}$ for $30 \mathrm{~min}$ as described for FRAC in C6 glioma cells (see below). Cells were lysed and lysates processed as previously described (Moutin et al., 2014) using GFP-Trap-A (ChromoTek) in place of RFP-Trap-A. After washing, the solid phase was incubated in Laemmli buffer at $60^{\circ} \mathrm{C}$ and protein samples resolved by PAGE on a $10 \%$ gel, transferred onto nitrocellulose sheet and subjected to immunoblotting using anti-kif5 antibody (1/2000, H2, MAB1614, Chemicon Int., Millipore) and anti-snap23 (1/2000, Synaptic Systems or Abcam) for $2 \mathrm{~h}$. Blots were then washed three times with PBS containing 0.1\% Tween-20 (PBST) and incubated with donkey anti-rabbit or anti-mouse peroxidaseconjugated antibodies (1/2000, Rockland) for $1 \mathrm{~h}$. Blots were 
washed five times with PBST and proteins were visualized with ECL Westen blotting detection reagent (Amersham, GE Healthcare Life Sciences) on ChemiDoc Imaging System (Bio-Rad).

\section{BRET measurements}

We have previously described BRET measurements in cell population (Perroy et al., 2004; Raynaud et al., 2013). Briefly, coexpression of mGlu1a-Rluc8 and YFP-SNAP23 or YFP-Kif5 in neuroblastoma cells was monitored using a spectrophotometric plate reader (MITHRAS LB 940, Berthold Technologies). BRET signal was plotted as a function of total fluorescence over total luminescence signal, and the ratio used as an index of the concentration of YFP-tagged over Rluc8-tagged proteins. Co-expression of increasing concentrations of YFP-tagged proteins with a constant level of Rluc8-tagged proteins gives rise to a hyperbolical increase and saturation in BRET signal, only if the two proteins specifically interact. Random collisions between tagged proteins gave a linear and non-saturating signal (bystander BRET), indicating non-specific protein-protein interactions.

\section{Fluorescence recovery after cleavage}

The mGlu1a receptor was tagged on its extracellular $\mathrm{N}$-terminal domain with a thrombin cleavable tag fused to YFP protein. The recombinant protein was transfected in C6 glioma cell line, and $24 \mathrm{~h}$ later cells were washed twice with DMEM medium (ref 61965, Thermo Fisher Scientific), supplemented with $10 \mathrm{mM}$ HEPES and $1 \mathrm{mg} / \mathrm{ml}$ chicken egg albumin (Sigma). Cells were then incubated at $20^{\circ} \mathrm{C}$ in the same medium to block post-Golgi traffic, followed by thrombin treatment $(5 \mathrm{U} / \mathrm{ml}$, Calbiochem) (Rosenberg et al., 2001) to cleave the external N-terminal YFP tag of cell surface mGlu1a receptors. After extensive washing, cells were returned to their initial medium at $37^{\circ} \mathrm{C}$ for cell surface receptor recovery. Cells were then washed and fixed at different time periods and quantification of cell surface receptor was determined using rabbit polyclonal anti-GFP antibodies (Invitrogen). For quantitative measurements, coverslips were transferred in 24-well microplates and processed for in-cell western assay (Odyssey, LI-COR Biosciences). Briefly, cells were blocked with gelatin, washed, incubated with anti-GFP antibodies, washed again and finally incubated with IRDye800CW-conjugated donkey anti-rabbit polyconal antibodies (Rockland). Infrared fluorescence was determined using the $700 \mathrm{~nm}$ channel of the Odyssey Infrared Imaging System (LI-COR Biosciences). Fluorescence intensity was measured using Image J software from circular gates placed in the middle of the wells. Background values measured in wells containing mocktransfected cells were subtracted from the signal. All measurements were performed in triplicate and expressed as mean \pm SEM.

\section{Rapalog-mediated protein heterodimerization}

Conventional kinesin1 (Kif5) rigor mutant tightly bound to microtubules in C6 glioma cells (data not shown) has been previously described for mouse fibroblast L cells (Nakata and Hirokawa, 1995). Presently, we replaced the tail of the protein, which binds the cargo, by FKBP-tagged or not with mcherry (Kif5 cargoless/FKBP and Kif5 cargoless/mcherry-FKBP). The
FRB-tagged protein consisted of CBD from Kif5 tail (FRB-mcherryCBD or FRB-YFP-CBD) and YFP-SNAP23 (FRB/YFP-SNAP23). Twenty-four hours after cell transfection, heterodimerization of the FRB-tagged and FKBP-tagged proteins was induced by the addition of $1 \mu \mathrm{M}$ heterodimerizer AP21967 (Ariad Pharmaceuticals), a rapamycin analog, for 15-60 min to drive the protein complex onto microtubules. Control (not treated with AP21967) and treated cells were then fixed and processed for microscopy.

\section{Microscopy}

Fixed cell images were acquired on a Zeiss Axiovert 200 TV inverted microscope equipped with a software-driven filter wheel, a $63 \times$ oil immersion objective [1.3 numerical aperture (NA); Zeiss], or a $25 \times$ oil immersion objective (0.8 NA; Zeiss). A MicroMax 1300 charge-device camera (Princeton Instruments) driven by MetaMorph imaging software (version 4.17; Universal Imaging Corporation) was used for cell imaging. Filter sets (Zeiss) for YFP-tagged proteins were $500 \pm 20$ and $535 \pm 30 \mathrm{~nm}$ band pass filters for excitation and emission, respectively. For mcherry-tagged proteins, we used filters (Omega Optical) with a $555 \pm 50 \mathrm{~nm}$ band pass and a $625 \pm 50 \mathrm{~nm}$ for excitation and emission, respectively. $Z$ series of images were acquired (31 planes) from $-3 \mu \mathrm{m}$ to $+3 \mu \mathrm{m}$ to image cells. An equatorial plane was selected from each series.

Imaging of live cells was performed on the same Zeiss setup. A homemade temperature-regulated chamber was used at $34^{\circ} \mathrm{C}$ to slightly reduce vesicle motion. For time-lapse imaging, exposure times were 300-500 ms for YFP-tagged proteins, 0.5-1 sec for mcherry-tagged proteins. Images from the MicroMax camera were converted to TIFF files that were edited using Image) 1.47 ( NIH Image) and compiled into QuickTime (Apple Computers) movies.

\section{Supplementary material}

Supplementary material is available at Journal of Molecular Cell Biology online.

\section{Acknowledgements}

Mass spectrometry experiments were carried out using facilities of the Functional Proteomics Platform of Montpellier and BRET experiments using facilities of Arpege pharmacological screening platform, at the Institute of Functional Genomics.

\section{Funding}

This work was supported by the European Research Council (ERC) under the European Union's Horizon 2020 research and innovation programme (grant agreement no. 646788 to J.P.), the Agence Nationale de la Recherche (ANR-13-JSV4-0005-01 to J.P.), and the Région Languedoc-Roussillon (Chercheur d'Avenir 146090 to J.P.).

Conflict of interest: none declared.

\section{References}

Aiba, A., Chen, C., Herrup, K., et al. (1994a). Reduced hippocampal long-term potentiation and context-specific deficit in associative learning in mGluR1 mutant mice. Cell 79, 365-375. 
Aiba, A., Kano, M., Chen, C., et al. (1994b). Deficient cerebellar long-term depression and impaired motor learning in mGluR1 mutant mice. Cell 79, 377-388.

Ango, F., Pin, J.P., Tu, J.C., et al. (2000). Dendritic and axonal targeting of type 5 metabotropic glutamate receptor is regulated by homer1 proteins and neuronal excitation. J. Neurosci. 20, 8710-8716.

Bindea, G., Mlecnik, B., Hackl, H., et al. (2009). ClueGO: a Cytoscape plug-in to decipher functionally grouped gene ontology and pathway annotation networks. Bioinformatics 25, 1091-1093.

Brabet, P., Pantaloni, C., Bockaert, J., et al. (1991). Metabolism of two Go alpha isoforms in neuronal cells during differentiation. J. Biol. Chem. 266, 12825-12828.

Castillo, C.A., Leon, D.A., Ballesteros-Yanez, I., et al. (2010). Glutamate differently modulates metabotropic glutamate receptors in neuronal and glial cells. Neurochem. Res. 35, 1050-1063.

Chan, W.Y., Soloviev, M.M., Ciruela, F., et al. (2001). Molecular determinants of metabotropic glutamate receptor 1B trafficking. Mol. Cell. Neurosci. 17, 577-588.

Choi, K.Y., Chung, S., and Roche, K.W. (2011). Differential binding of calmodulin to group I metabotropic glutamate receptors regulates receptor trafficking and signaling. J. Neurosci. 31, 5921-5930.

Cox, J., and Mann, M. (2008). MaxQuant enables high peptide identification rates, individualized p.p.b.-range mass accuracies and proteome-wide protein quantification. Nat. Biotechnol. 26, 1367-1372.

Cox, J., Neuhauser, N., Michalski, A., et al. (2011). Andromeda: a peptide search engine integrated into the MaxQuant environment. J. Proteome Res. 10, 1794-1805.

Das, S.S., and Banker, G.A. (2006). The role of protein interaction motifs in regulating the polarity and clustering of the metabotropic glutamate receptor mGluR1a. J. Neurosci. 26, 8115-8125.

Diefenbach, R.J., Diefenbach, E., Douglas, M.W., et al. (2002). The heavy chain of conventional kinesin interacts with the SNARE proteins SNAP25 and SNAP23. Biochemistry 41, 14906-14915.

Duc, C., and Catsicas, S. (1995). Ultrastructural localization of SNAP-25 within the rat spinal cord and peripheral nervous system. J. Comp. Neurol. 356, 152-163.

Ferraguti, F., Crepaldi, L., and Nicoletti, F. (2008). Metabotropic glutamate 1 receptor: current concepts and perspectives. Pharmacol. Rev. 60, 536-581.

Giau, R., Carrette, J., Bockaert, J., et al. (2005). Constitutive secretion of protease nexin- 1 by glial cells and its regulation by G-protein-coupled receptors. J. Neurosci. 25, 8995-9004.

Guillaud, L., Setou, M., and Hirokawa, N. (2003). KIF17 dynamics and regulation of NR2B trafficking in hippocampal neurons. J. Neurosci. 23, 131-140.

Hannan, A.J., Blakemore, C., Katsnelson, A., et al. (2001). PLC-beta1, activated via mGluRs, mediates activity-dependent differentiation in cerebral cortex. Nat. Neurosci. 4, 282-288.

Hirokawa, N., Niwa, S., and Tanaka, Y. (2010). Molecular motors in neurons: transport mechanisms and roles in brain function, development, and disease. Neuron 68, 610-638.

Hoerndli, F.J., Maxfield, D.A., Brockie, P.J., et al. (2013). Kinesin-1 regulates synaptic strength by mediating the delivery, removal, and redistribution of AMPA receptors. Neuron 80, 1421-1437.

Ichise, T., Kano, M., Hashimoto, K., et al. (2000). mGluR1 in cerebellar Purkinje cells essential for long-term depression, synapse elimination, and motor coordination. Science 288, 1832-1835.

Jahn, R., and Scheller, R.H. (2006). SNAREs_engines for membrane fusion. Nat. Rev. Mol. Cell Biol. 7, 631-643.

Jenkins, B., Decker, H., Bentley, M., et al. (2012). A novel split kinesin assay identifies motor proteins that interact with distinct vesicle populations. J. Cell Biol. 198, 749-761.

Kenny, P.J., and Markou, A. (2004). The ups and downs of addiction: role of metabotropic glutamate receptors. Trends Pharmacol. Sci. 25, 265-272.
Kitano, J., Kimura, K., Yamazaki, Y., et al. (2002). Tamalin, a PDZ domaincontaining protein, links a protein complex formation of group 1 metabotropic glutamate receptors and the guanine nucleotide exchange factor cytohesins. J. Neurosci. 22, 1280-1289.

Ledonne, A., Nobili, A., Latagliata, E.C., et al. (2015). Neuregulin 1 signalling modulates mGluR1 function in mesencephalic dopaminergic neurons. Mol. Psychiatry 20, 959-973.

Lin, T.B., Lai, C.Y., Hsieh, M.C., et al. (2015). VPS26A-SNX27 interactiondependent mGluR5 recycling in dorsal horn neurons mediates neuropathic pain in rats. J. Neurosci. 35, 14943-14955.

Lin, R.C., and Scheller, R.H. (2000). Mechanisms of synaptic vesicle exocytosis. Annu. Rev. Cell Dev. Biol. 16, 19-49.

Luis Albasanz, J., Fernandez, M., and Martin, M. (2002). Internalization of metabotropic glutamate receptor in $\mathrm{C} 6$ cells through clathrin-coated vesicles. Brain Res. Mol. Brain Res. 99, 54-66.

Luscher, C., and Huber, K.M. (2010). Group 1 mGluR-dependent synaptic long-term depression: mechanisms and implications for circuitry and disease. Neuron 65, 445-459.

Moutin, E., Compan, V., Raynaud, F., et al. (2014). The stoichiometry of scaffold complexes in living neurons-DLC2 functions as a dimerization engine for GKAP. J. Cell Sci. 127, 3451-3462.

Nakata, T., and Hirokawa, N. (1995). Point mutation of adenosine triphosphate-binding motif generated rigor kinesin that selectively blocks anterograde lysosome membrane transport. J. Cell Biol. 131, 1039-1053.

Noel, J., Ralph, G.S., Pickard, L., et al. (1999). Surface expression of AMPA receptors in hippocampal neurons is regulated by an NSF-dependent mechanism. Neuron 23, 365-376.

Olsen, J.V., de Godoy, L.M., Li, G., et al. (2005). Parts per million mass accuracy on an Orbitrap mass spectrometer via lock mass injection into a C-trap. Mol. Cell. Proteomics 4, 2010-2021.

Orchard, S., Ammari, M., Aranda, B., et al. (2014). The MlntAct projectIntAct as a common curation platform for 11 molecular interaction databases. Nucleic Acids Res. 42, D358-D363.

Osten, P., Srivastava, S., Inman, G.J., et al. (1998). The AMPA receptor GluR2 $C$ terminus can mediate a reversible, ATP-dependent interaction with NSF and alpha- and beta-SNAPs. Neuron 21, 99-110.

Oyler, G.A., Higgins, G.A., Hart, R.A., et al. (1989). The identification of a novel synaptosomal-associated protein, SNAP-25, differentially expressed by neuronal subpopulations. J. Cell Biol. 109, 3039-3052.

Perroy, J., Pontier, S., Charest, P.G., et al. (2004). Real-time monitoring of ubiquitination in living cells by BRET. Nat. Methods 1, 203-208.

Ravichandran, V., Chawla, A., and Roche, P.A. (1996). Identification of a novel syntaxin- and synaptobrevin/VAMP-binding protein, SNAP-23, expressed in non-neuronal tissues. J. Biol. Chem. 271, 13300-13303.

Raynaud, F., Janossy, A., Dahl, J., et al. (2013). Shank3-Rich2 interaction regulates AMPA receptor recycling and synaptic long-term potentiation. J. Neurosci. 33, 9699-9715.

Rosenberg, M., Meier, J., Triller, A., et al. (2001). Dynamics of glycine receptor insertion in the neuronal plasma membrane. J. Neurosci. 21, 5036-5044.

Setou, M., Nakagawa, T., Seog, D.H., et al. (2000). Kinesin superfamily motor protein KIF17 and mLin-10 in NMDA receptor-containing vesicle transport. Science 288, 1796-1802.

Shannon, P., Markiel, A., Ozier, O., et al. (2003). Cytoscape: a software environment for integrated models of biomolecular interaction networks. Genome Res. 13, 2498-2504.

Suh, Y.H., Terashima, A., Petralia, R.S., et al. (2010). A neuronal role for SNAP-23 in postsynaptic glutamate receptor trafficking. Nat. Neurosci. 13 338-343.

Tao-Cheng, J.H., Du, J., and McBain, C.J. (2000). Snap-25 is polarized to axons and abundant along the axolemma: an immunogold study of intact neurons. J. Neurocytol. 29, 67-77.

Thouvenot, E., Urbach, S., Dantec, C., et al. (2008). Enhanced detection of CNS cell secretome in plasma protein-depleted cerebrospinal fluid. J. Proteome Res. 7, 4409-4421. 
Viwatpinyo, K., and Chongthammakun, S. (2009). Activation of group I metabotropic glutamate receptors leads to brain-derived neurotrophic factor expression in rat C6 cells. Neurosci. Lett. 467, 127-130.

Washbourne, P., Bennett, J.E., and McAllister, A.K. (2002a). Rapid recruitment of NMDA receptor transport packets to nascent synapses. Nat. Neurosci. 5, 751-759.

Washbourne, P., Liu, X.B., Jones, E.G., et al. (2004). Cycling of NMDA receptors during trafficking in neurons before synapse formation. J. Neurosci. 24, 8253-8264.
Washbourne, P., Thompson, P.M., Carta, M., et al. (2002b). Genetic ablation of the t-SNARE SNAP-25 distinguishes mechanisms of neuroexocytosis. Nat. Neurosci. 5, 19-26.

Wu, H., Nash, J.E., Zamorano, P., et al. (2002). Interaction of SAP97 with minus-end-directed actin motor myosin VI. Implications for AMPA receptor trafficking. J. Biol. Chem. 277, 30928-30934.

Zhou, L., Yang, D., Wang, D.J., et al. (2015). Numb deficiency in cerebellar Purkinje cells impairs synaptic expression of metabotropic glutamate receptor and motor coordination. Proc. Natl Acad. Sci. USA 112, 15474-15479. 